\title{
THE FADING OF YOUNG STELLAR POPULATIONS AND THE LUMINOSITY FUNCTIONS OF DWARF, IRREGULAR, AND STARBURST GALAXIES
}

\author{
David W. Hogg and E. S. Phinney \\ Theoretical Astrophysics, California Institute of Technology, Mail Code 130-33, Pasadena, CA 91125; hogg@tapir.caltech.edu, esp@tapir.caltech.edu \\ Received 1997 June 2; accepted 1997 August 19; published 1997 September 22
}

\begin{abstract}
Dwarf, irregular, and infrared-luminous starburst galaxies are all known to have "steep" luminosity functions with faint-end behavior roughly $\phi(L) \propto L^{-1.8}$. This form is exactly what is expected if the luminosities of these objects fade with time as $L \propto t^{-1.3}$, because the objects spend more time at low luminosities than high, even if they form with a wide range of initial masses. Models of young stellar populations show this fading behavior when the star formation has occurred in a single, short, recent burst. Steep luminosity functions therefore do not require steep mass functions if the galaxies are powered by fading bursts. The local galaxy $\mathrm{H} \alpha$ luminosity function-which is less steep than $L^{-1.8}$-is also well fitted by this mechanism, because ionizing photon flux fades much more quickly than broadband optical luminosity. An age-luminosity relation and a wavelength dependence of the luminosity function are both predicted. In the context of this mechanism, the slope of the luminosity function provides a constraint on the stellar initial mass function in the bursts.
\end{abstract}

Subject headings: galaxies: luminosity function, mass function — galaxies: starburst galaxies: stellar content — infrared: galaxies — stars: luminosity function, mass function

\section{INTRODUCTION}

While the normal field galaxy luminosity function (GLF) $\phi(L)$ (number density per unit luminosity) or $\phi(\log L)$ (number density per unit log luminosity) is "flat" in optical bandpasses at the faint end, i.e., $\phi(L) \propto L^{-1}$ or $\phi(\log L)=$ constant (Efstathiou, Ellis, \& Peterson 1988; Loveday et al. 1992; Mobasher, Sharples, \& Ellis 1993; Marzke, Huchra, \& Geller 1994b; Lin et al. 1996, 1997; Gardner et al. 1997; Ratcliffe et al. 1997), many studies have found that objects in which the luminosity is thought to be dominated by young stars show a "steep" GLF, with roughly $\phi(L) \propto L^{-1.8}$ at the faint end. Parameterizing $\phi(L) \propto L^{\alpha}$, the $60 \mu \mathrm{m}$ GLF from the IRAS bright galaxy sample appears to show $\alpha=-1.8$ at the faint end (Soifer et al. 1987; although see Saunders et al. 1990), despite the fact that these same objects lie in the flat part of the optical GLF. The $60 \mu \mathrm{m}$ luminosity is thought to originate in dust heated by the radiation from young stars at ultraviolet wavelengths, where dusty galaxies are optically thick. Although the faint end of the optical GLF is flat, there may also be a small "upturn" at the very faintest end, around absolute magnitude $M_{B}=-16$ mag (Marzke et al. 1994b; Driver \& Phillips 1996; Loveday 1997), which is explained by a luminosity function with $\alpha=-1.8$ among dwarf (i.e., low-luminosity) galaxies. In the case of the CfA survey, the upturn can be explained entirely by the luminosity function of the Sm-Im galaxies (identified on the basis of morphology), which show $\alpha=-1.87 \pm 0.2$ (Marzke et al. 1994a). Local galaxies spectrally classified as strongly or recently star-forming also show a steep luminosity function (Heyl et al. 1997). A steep upturn at the faint end of the GLF is observed for dwarf galaxies in rich clusters with $\alpha$ ranging from -1.4 to -2.2 (Sandage, Binggeli, \& Tammann 1985; Driver et al. 1994b; Bernstein et al. 1995; De Propris et al. 1995; Lobo et al. 1996; Wilson et al. 1997). A recent measurement of the luminosity function of dwarf galaxies or "knots" formed in the tidal tails of merging galaxies finds $\alpha=$ $-1.75 \pm 0.27$ in the $R$ band for these objects (Hunsberger, Charlton, \& Zaritsky 1996). Compact "super-star clusters" observed in the vicinity of starburst galaxies or galaxy mergers and interpreted as the progenitors of globular clusters show a luminosity function consistent with $\alpha=-1.8$, although the numbers are small (Lutz 1991; Holtzman et al. 1992; Whitmore et al. 1993; Conti \& Vacca 1994). Finally, a luminosity function with faint-end behavior $\alpha \approx-1.8$ is often invoked as a natural explanation of faint galaxy counts and redshift distributions (Broadhurst, Ellis, \& Shanks 1988; Eales 1993; Koo, Gronwall, \& Bruzual 1993; Driver et al. 1994a; Treyer \& Silk 1994; Metcalfe et al. 1995; Smail et al. 1995; Lilly et al. 1995; Ellis et al. 1996). In these studies, the steep GLF is largely required to account for the large numbers of faint blue galaxies, which are mainly irregulars (Glazebrook et al. 1995b; Driver, Windhorst, \& Griffiths 1995; Abraham et al. 1996) and are thought to have luminosities dominated by young stars.

The steep faint end of the GLF is usually attributed to a steep underlying galaxy mass function. A steep mass function at small halo mass $M_{h}$ is natural for cold and mixed dark matter models. In the Press \& Schechter (1974) formalism, on small mass scales $\phi\left(M_{h}\right) d M_{h} \propto M_{h}^{-[(9-n) / 6]} d M_{h}$, where the postrecombination power spectrum of density fluctuations has $P(k) \propto$ $k^{n}$, with $n \rightarrow-3$ for adiabatic fluctuations on small scales. Thus, $\phi\left(M_{h}\right) d M_{h} \propto M_{h}^{-2} d M_{h}$. This has been amply verified by numerical simulation for both cold (Brainerd \& Villumsen 1992) and mixed (Ma \& Bertschinger 1994) dark matter halos. However, the ejection of gas by early generations of stars in shallow potential wells implies that the mass converted to stars rises faster than linearly with $M_{h}$, so for identical stellar populations, the GLF should be shallower than the halo mass function (see Silk \& Wyse 1993 for a review). Furthermore, in the IR-luminous galaxy sample, the large scatter in optical-IR colors (Soifer et al. 1987) and the lack of correlation between IR luminosity and galaxy mass inferred from rotation curves (Lehnert \& Heckman 1996) suggest that the starburst GLF is not strongly tied to the host galaxy mass function.

In this Letter, we remark that there is a natural mechanism that ensures a steep GLF among young objects. Even if the galaxy mass function (where now by "galaxy mass" is meant "the mass of that part of the galaxy's baryonic mass that is turned into stars") is flat, a GLF of roughly the form $\phi(L) \propto$ $L^{-1.8}$ will be observed among any population of objects whose 
TABLE 1

EXPONENTS OF LUMINOSITY DECAY ${ }^{\mathrm{a}}$

\begin{tabular}{ccccccc}
\hline \hline Reference & $x, Z$ & $\zeta_{\text {bol }}$ & $\zeta_{U}$ & $\zeta_{B}$ & $\zeta_{V}$ & $\zeta_{K}$ \\
\hline $1^{\mathrm{b}} \ldots \ldots \ldots$ & $x=1.35, Z=Z_{\odot}$ & $\ldots$ & -1.2 & -1.0 & -0.9 & -0.7 \\
$2^{\mathrm{c}} \ldots \ldots \ldots$ & $x=1.35, Z=0.1 Z_{\odot}$ & -1.4 & -1.2 & -1.0 & -1.0 & -0.5 \\
$2^{\mathrm{c}} \ldots \ldots \ldots$ & $x=2.3, Z=0.1 Z_{\odot}$ & -0.7 & -0.5 & -0.4 & -0.3 & -0.0 \\
\hline
\end{tabular}

${ }^{a}$ See eq. (3).

${ }^{\text {b }}$ Bruzual \& Charlot 1993. Luminosities are fitted over the age range $10^{7}$ $\mathrm{yr}<t<10^{10.4} \mathrm{yr}$. The IMF slope $x$ (eq. [1]) is relevant only for stars with masses greater than $1 M_{\odot}$.

${ }^{c}$ Leitherer \& Heckman 1995. Luminosities are fitted over the age range $10^{6.5} \mathrm{yr}<t<10^{8.5} \mathrm{yr}$. The IMF slope $x$ (eq. [1]) is relevant only for stars with masses greater than $2 M_{\odot}$.

luminosities are dominated by light from short, recent bursts of star formation with a Salpeter-like initial mass function (IMF). This is because their luminosities decrease with time in such a way that they spend more time (and are therefore more numerous) at low luminosities than at high luminosities. This kind of mechanism underlies the theoretical explanation of the $60 \mu \mathrm{m}$ GLF by Scoville \& Soifer (1991) and a discussion of the super-star cluster luminosity function by Meurer (1995). An important feature of this mechanism is that steep mass functions are not required to explain steep luminosity functions.

\section{EXPOSITION}

The luminosity $L$ of a main-sequence star of mass $M>$ $1 M_{\odot}$ scales as $L \propto M^{\eta}$, where $\eta \approx 3.9$ (Kippenhahn \& Weigert 1990 , p. 209). Thus, the main-sequence lifetime $\tau \propto M / L \propto$ $M^{1-\eta}$. Let an ensemble of stars be formed in a single burst with IMF $\phi(M) d M \propto M^{-(1-x)} d M$ (where $x=1.35$ is the Salpeter slope). The stages of stellar evolution beyond the main sequence have lifetimes and an integrated consumption of nuclear fuel less than or comparable to the main-sequence values (Renzini \& Buzzoni 1986). Thus, for $x<\eta$, the total luminosity of the evolving ensemble is dominated by stars just leaving the main sequence, with mass $M=M_{\mathrm{turn}}(t)$ :

$$
\left.L_{\mathrm{Tot}} \simeq C \int_{M_{\odot}}^{M_{\mathrm{turn}}}(t) \phi(M) L(M) d M \simeq \frac{C}{\eta-x} M \phi(M) L(M)\right|_{M_{\mathrm{turn}(t)},},
$$

where $C$ is a factor of order 2 (Renzini \& Buzzoni 1986), which accounts for the post-main-sequence fuel consumption. Since $M_{\mathrm{turn}}(t)$ is defined by $t=\tau\left(M_{\mathrm{turn}}\right)$, we have for $x<\eta$,

$$
L_{\mathrm{Tot}} \propto t^{\zeta}, \quad \zeta=\frac{x-\eta}{\eta-1},
$$

so in this approximation $\zeta \approx-0.9$ for a Salpeter IMF. The luminosity in a particular wavelength band $X$ will have a somewhat different dependence on age than the bolometric luminosity, depending on bolometric correction, but since stars consume about as much fuel in the (red) post-main sequence as during the (blue) main sequence, the dependence in optical and near-IR bands will not be vastly different from that of the bolometric luminosity.

If in wavelength band $X$ an object has luminosity $L_{X}$, which varies with time $t$ since its birth as

$$
L_{X} \propto t^{5 x}
$$

and the object is observed at a random time after its birth, then the probability that it is measured to have luminosity $L_{X}$ is proportional to $\left(d L_{X} / d t\right)^{-1} \propto L_{X}^{\left(1 / \zeta_{X}\right)-1}$. Therefore, a population of identical objects of this type born at a fairly uniform rate over a time interval will appear to have a luminosity function with faint-end slope $\alpha_{X}=\left(1 / \zeta_{X}\right)-1$ despite being intrinsically identical. The luminosity function is produced by a spread in ages rather than a distribution of intrinsic properties. In order to make a luminosity function with $\alpha \approx-1.8, \zeta \approx-1.3$ is required.

In any realistic scenario, the objects will have a distribution of intrinsic sizes or starburst masses. Consider objects with luminosities $L_{X}=M \Lambda_{X}(t)$, where $M$ is the mass of stars created in the starburst, the objects are selected from a distribution $\phi(M)$ of starburst masses, and $\Lambda_{X}(t) \propto t^{\zeta_{X}}$ is the light per unit mass in wavelength band $X$ as a function of time. The faint end of the luminosity function will still show $\phi(L) \propto L^{(1 / \zeta)-1}$ as long as $\phi(M)$ is flatter than $\phi(M) \propto M^{(1 / \zeta)-1}$ (i.e., the exponent is less negative than $(1 / \zeta)-1)$ at the small-mass end and steeper than $\phi(M) \propto M^{(1 / /)-1}$ at the large-mass end. Both conditions hold if the "flat" Schechter function observed for the local GLF represents the intrinsic mass distribution for galaxies.

With models much more detailed than the scaling argument given at the beginning of this section, Leitherer \& Heckman
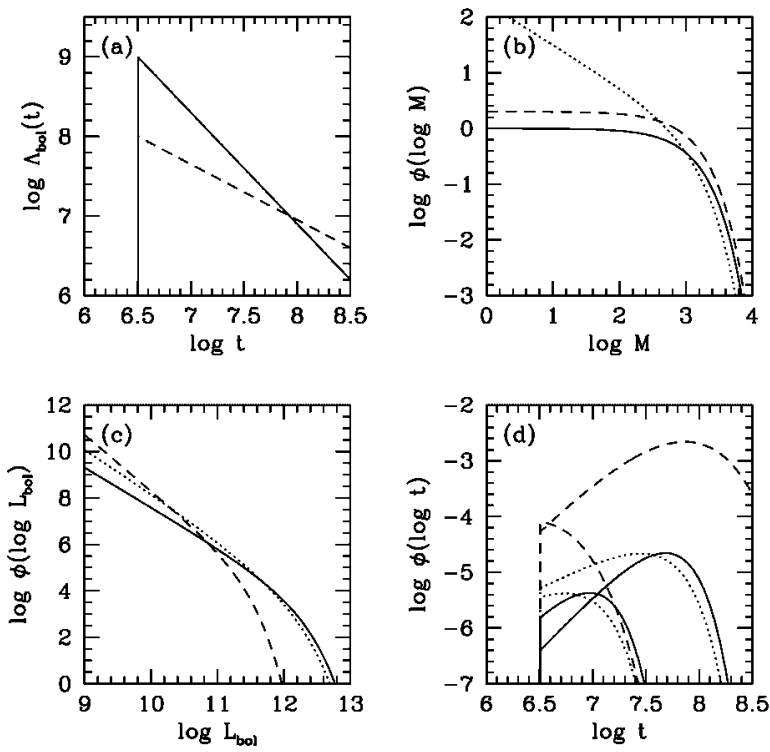

FIG. 1.-An illustration of the fading mechanism for the steep GLF presented in this Letter. Shown are (a) the bolometric fading laws $\Lambda_{\text {bol }}(t)$ (bolometric luminosity per unit mass in solar units) used, which are crude fits to Fig. 7 of Leitherer \& Heckman (1995); (b) the mass functions $\phi(\log M) \propto M \phi(M)$ (number per decade in mass), which are really the distributions of starburst masses, arbitrarily choosing $M^{*}=10^{6} M_{\odot} ;(c)$ the bolometric luminosity functions $\phi\left(\log L_{\mathrm{bol}}\right) \propto L_{\mathrm{bol}} \phi\left(L_{\mathrm{bol}}\right)$ (number per decade in luminosity) produced by the fading and mass functions; and $(d)$ the age function $\phi(\log t) \propto t \phi(t)$ (number per decade in age) for objects at luminosity $L_{\text {bol }}=10^{11.5} L_{\odot}$ (bottom curves) and $L_{\text {bol }}=10^{10.5} L_{\odot}$ (top curves). Three models are shown, one with $\zeta=$ -1.4 and $\beta=-1.0$ (solid line), $\zeta=-1.4$ and $\beta=-1.75$ (dotted line), and $\zeta=-0.7$ and $\beta=-1.0$ (dashed line). See text for symbol definitions. Mass, luminosity, and age functions all have arbitrary normalizations; it is not meaningful to compare relative heights of two different curves on the same plot. 
(1995) and Bruzual \& Charlot (1993) predict the fading with time of young stellar populations in a number of photographic bands and for several models of the stellar IMF and metallicity, to which power laws have been fitted. The results are given in Table 1. The exponents in the ultraviolet and optical, for a Salpeter IMF (slope $x=1.35$ ) are in the range $-1.4<\zeta<$ -0.9 , which lead to luminosity functions with $-2.1<\alpha<$ -1.7. A Miller-Scalo IMF (slope $x=2.3$ ) produces slower fading and therefore steeper (more negative $\alpha$ ) luminosity functions. Thus, if the luminosity function of faint galaxies is indeed produced by fading of young populations, then the slope of the luminosity function can be used to constrain the stellar IMF. The different luminosity function slopes observed in different clusters (Binggeli, Sandage \& Tammann 1985; Driver et al. 1994b; Bernstein et al. 1995; De Propris et al. 1995; Lobo et al. 1996; Wilson et al. 1997) could reflect an environmental dependence in the stellar IMF, although the statistics are small enough that the various studies appear to be at least marginally consistent with one another.

In addition, the evolution of the rate $\Gamma_{i}$ of production of ionizing photons (i.e., photons shortward of $912 \AA$ ) is predicted (Leitherer \& Heckman 1995), with roughly $\Gamma_{i} \propto t^{-4.9}$. If the strength of $\mathrm{H} \alpha$ emission is proportional to $\Gamma_{i}$, then this fading behavior naturally produces an $\mathrm{H} \alpha$ luminosity function with faint-end slope $\alpha=-1.2$, consistent with a recent measurement of $\alpha=-1.3 \pm 0.2$ for the local H $\alpha$ GLF (Gallego et al. 1995). form

As an illustration, Figure 1 shows (a) fading laws of the

$$
\Lambda_{\mathrm{bol}}(t)= \begin{cases}0 & \text { for } t<t_{0} \\ \Lambda_{0}\left(\frac{t}{t_{0}}\right)^{\zeta} & \text { for } t>t_{0}\end{cases}
$$

with $\zeta=-1.4$ and -0.7 , which serve as approximations to Figure 7 of Leitherer \& Heckman (1995); (b) distributions of starburst masses $\phi(\log M)$ (number per decade in mass) of the Schechter (1976) form,

$$
\phi(\log M) \propto M \phi(M) \propto\left(\frac{M}{M^{*}}\right)^{\beta+1} e^{-M / M^{*}}
$$

where $\beta$ sets the slope of the mass function at the low-mass end, and $M^{*}$ (fixed at $10^{3} M_{\odot}$ for definiteness) sets the highmass cutoff; (c) the resulting luminosity functions $\phi(\log L) \propto L \phi(L)$ (number per decade in luminosity) under the assumption that the populations are observed at random ages as they fade; and $(d)$ the age distributions $\phi(\log t)$ (number per decade in age) at two different luminosity levels $\left(10^{11.5}\right.$ and $10^{10.5} L_{\odot}$ in this case). This shows that the luminosity function has the predicted power-law form even if there is a broad distribution of starburst masses. It also shows that the distribution of ages can be broad but does indeed get older as the objects get fainter. The age function is broadest in the cases in which the mass function is almost as steep as the luminosity function.

As an illustration, the upturn in the local GLF at absolute magnitude $M_{B} \approx-16\left(H_{0}=100 \mathrm{~km} \mathrm{~s}^{-1} \mathrm{Mpc}^{-1}\right)$ found by Marzke et al. (1994b) can be analyzed in terms of the fading mechanism. The GLF has amplitude $\phi(\log L) \approx 0.09 \mathrm{Mpc}^{-3}$ at absolute magnitude $M_{B}=-16$ or luminosity $L_{B}=3.7 \times$
$10^{8} L_{\odot}$ and slope $\alpha \approx-2$ faintward. To order of magnitude, by dimensional analysis,

$$
\begin{aligned}
\phi\left(\log L_{B}\right) & \equiv(\ln 10) L_{B} \frac{d N}{d V d L_{B}} \\
& =(\ln 10) L_{B} \frac{d N}{d V d t} \frac{1}{M_{\mathrm{typ}}}\left|\frac{d \Lambda_{B}}{d t}\right|^{-1},
\end{aligned}
$$

where $d N /(d V d t)$ is the birthrate density (number per unit time per unit volume), $M_{\text {typ }}$ is the typical starburst mass, and $d \Lambda_{B} / d t$ is the rate of change of the light-to-mass ratio in the $B$ band, evaluated at the light-to-mass ratio $\Lambda_{B}=L_{B} / M_{\mathrm{typ}}$ at which starbursts of mass $M_{\mathrm{typ}}$ have luminosity $L_{B}$. At this value,

$$
\frac{d \Lambda_{B}}{d t}=\frac{\zeta_{B} \Lambda_{0}}{t_{0}}\left(\frac{t}{t_{0}}\right)^{\zeta_{B}-1}=\frac{\zeta_{B} \Lambda_{0}}{t_{0}}\left(\frac{L_{B}}{M_{\mathrm{typ}} \Lambda_{0}}\right)^{1-\left(1 / \zeta_{B}\right)}
$$

where $\Lambda_{0}=5.8 \times 10^{8} L_{\odot} / M_{\odot}, t_{0}=3 \times 10^{6} \mathrm{yr}$, and $\zeta_{B}=$ -1.0 are the appropriate values for the fading-law parameters in equation (4) for the $B$ band (Leitherer \& Heckman 1995). Plugging in these values, a relationship between the birthrate density and the typical mass is derived,

$$
\begin{aligned}
\frac{d N}{d V d t} & =\frac{\left|\zeta_{B}\right| \phi\left(\log L_{B}\right)}{(\ln 10) t_{0}}\left(\frac{M_{\mathrm{typ}} \Lambda_{0}}{L_{B}}\right)^{1 / \zeta_{B}} \\
& =8.3 \times 10^{-3}\left(\frac{M_{\mathrm{typ}}}{10^{3} M_{\odot}}\right)^{-1} \mathrm{Mpc}^{-3} \mathrm{Gyr}^{-1}
\end{aligned}
$$

for $H_{0}=100 \mathrm{~km} \mathrm{~s}^{-1} \mathrm{Mpc}^{-1}$. Note that the model is not unique because neither the birthrate density nor the typical mass can be determined from the above information alone, only a relationship between the two.

Of course, this fading mechanism requires a fairly uniform birthrate. In the extreme, if in some population all starbursts occurred simultaneously at some moment in the past, no spread of ages would be observed, and the luminosities would be proportional only to the intrinsic sizes of the starbursts. The GLF would have nothing to do with the fading exponent $\zeta_{X}$ at all. If the birthrate is varying slowly with time, it will affect the final luminosity function shape. For instance, if the birthrate is decreasing with time, then there will be more old galaxies than young; therefore, more low-luminosity systems than would be predicted for constant birthrate and a steeper GLF will be observed (i.e., $\alpha$ will be more negative than $\left(1 / \zeta_{X}\right)-$ 1). Again, different slopes among, say, different galaxy cluster GLFs could indicate different dependences of starburst birthrate on time. However, over the small dynamic ranges (and hence age ranges) over which such GLFs have usually been measured, the assumption of relatively constant birthrate is not unreasonable.

If individual galaxies undergo multiple starbursts, then this fading mechanism will still ensure a steep GLF, as long as the sources are fading according to $\Lambda_{X}(t) \propto t^{\zeta x}$. For a multipleburst source this will be true at young ages, when the recently formed stars still dominate the light. However, at late times the underlying population of old stars from previous bursts will eventually become a significant, nonfading contribution to the luminosity. Thus, multiple-burst sources will show the steep GLF, but perhaps over a somewhat restricted range of age and therefore luminosity. Of course, because young stellar popu- 
lations are so much brighter per unit mass than old, this will not be a big restriction unless the sources burst very frequently (in which case the basic assumption of the model breaks down).

\section{PREDICTION}

This fading model for the faint end of the luminosity function makes three important predictions. The first prediction is that the bright members of the populations that show these steep luminosity functions should be mostly young. The distribution of ages and, in particular, the modal age $t_{m}\left(L_{X}\right)$ at each luminosity $L_{X}$ in band $X$ should obey the law $t_{m}\left(L_{X}\right) \propto L^{1 / 5 x}$. A correlation between dust temperature and luminosity is observed in IRAS galaxies (Miley, Neugebauer, \& Soifer 1985; Rieke \& Lebofsky 1986; Soifer et al. 1987). In models in which the far-IR emission is from dust heated by young stars, this relation can be interpreted as an age-luminosity relation, although it is also naturally produced if the dust is heated by other mechanisms (e.g., nuclear activity or continuous star formation). Spectra of $I R A S$ galaxies in the $\phi(L) \propto L^{-1.8}$ part of the $60 \mu \mathrm{m}$ luminosity function could be used to confirm this interpretation of the temperature-luminosity relation. In the optical an age-luminosity relation for a starburst can be converted into a color-luminosity relation; the expectation is that fainter galaxies will be redder, in possible contradiction to the evidence from some samples of extragalactic H II regions (Telles 1995) and faint galaxy counts (e.g., Koo \& Kron 1992). Again, spectra of nearby dwarf, irregular, or IR-luminous galaxies could in principle be used to determine a quantitative age-luminosity relation. Because the luminosities are determined more by age than by intrinsic properties, there ought to be little correlation between the luminosities and dynamical masses of the galaxies, as measured from rotation curves or velocity dispersions. Of course, the width of the age distribution at a given luminosity depends on the underlying starburst mass distribution discussed above. If the starburst mass distribution $\phi(m)$ is a Schechter function with faint-end slope $\beta$, then the width of the age distribution increases as $\beta \rightarrow \alpha=(1 / \zeta)-1$ (see Fig. $1 d$ ). (Recall that if $\beta<\alpha$, then the overall luminosity function will have faint-end slope $\beta$ rather than $\alpha$.) In principle, this effect could be used to constrain the distribution of starburst masses.

The luminosity function shape depends on the objects fading out of the sample with time, so the second prediction is that this mechanism can only explain the slope of the luminosity function over as many magnitudes, as the fading obeys the power law. This range appears to be at least 7.5 mag (factor of $10^{3}$ ) for the visible-band luminosities (Bruzual \& Charlot 1993) — unless the decay is interrupted by a subsequent burst of star formation, of course-but luminosity functions with steep faint-end slopes over a significantly greater range cannot be explained entirely by this mechanism. The largest dynamic range for which the slope has been measured so far is in the IRAS sample, which appears to show slope $\alpha=-1.8$ over a factor of roughly 300 in $60 \mu \mathrm{m}$ luminosity (Soifer et al. 1987). The luminosity functions of cluster galaxies will have to be measured to levels more than 3 mag fainter than current observational limits in order to exceed the allowed range.

The third prediction comes from the different slopes of $\Lambda(t)$ in different wavelength bands. These translate into different predicted luminosity function slopes $\alpha$. In general, the luminosity function is expected to become steeper ( $\alpha$ more negative) as the objects are observed in longer wavelength bands. This prediction is related to the color-luminosity relation predicted above and is easy to test with multiband imaging of dwarf galaxy populations. There is already some evidence in contradiction to this prediction for galaxy cluster GLFs (Wilson et al. 1997), and faint galaxy counts, which may be naturally explained with steep luminosity functions, become flatter, not steeper, with increasing wavelength (e.g., Koo \& Kron 1992). Of course the faint galaxy counts are difficult to interpret, since the observed objects are effectively integrated over a range of redshifts (and hence rest wavelengths) and there is the possibility that multiple distinct populations play comparable roles. It is encouraging that the $\mathrm{H} \alpha$ luminosity function (Gallego et al. 1995) is well-fitted by this mechanism; under this interpretation the $\mathrm{H} \alpha$ luminosity becomes more a measure of time since most recent starburst than a measure of star formation rate, as is conventionally assumed. Conclusions about the volume-averaged star formation rate of the local universe (Gallego et al. 1995) are not strongly affected by this change in interpretation, however, because such conclusions depend only on total emission of ionizing photons, not on whether or not the emission is simultaneous with or subsequent to the star formation.

\section{APPLICATION}

Finally, we emphasize that if the fading of short, recent bursts of star formation does indeed explain the faint-end slope of GLFs, then the measured slope becomes a strong constraint on the stellar IMF in these objects. Since other techniques (e.g., population synthesis models) often do not constrain the IMF uniquely in individual objects (e.g., Santos et al. 1995), the slope of the faint end of the GLF may become an extremely useful diagnostic for star and galaxy formation models.

We benefited from helpful comments from Lee Armus, Roger Blandford, Tim Heckman, Jeremy Heyl, Nick Scoville, Tom Soifer, and an anonymous referee. Claus Leitherer, Tim Heckman, and Jeff Goldader generously provided us with published data in electronic form. Support from the National Science Foundation is gratefully acknowledged (D. W. H. under AST-9529170 and E. S. P. under AST-9315455).

\section{REFERENCES}

Abraham, R. G., Tanvir, N. R., Santiago, B. X., Ellis, R. S., Glazebrook, K., \& van den Bergh, S. 1996, MNRAS, 279, L47

Bernstein, G. M., Nichol, R., Tyson, J. A., Ulmer, M. P., \& Wittman, D. 1995, AJ, 110, 1507

Binggeli, B., Sandage, A., \& Tammann, G. A. 1985, AJ, 90, 1681

Brainerd, T. G., \& Villumsen, J. V. 1992, ApJ, 394, 409

Broadhurst, T. J., Ellis, R. S., \& Shanks, T. 1988, MNRAS, 235, 827

Bruzual A., G., \& Charlot, S. 1993, ApJ, 405, 538

Conti, P. S., \& Vacca, W. D. 1994, ApJ, 423, L97

De Propris, R., Pritchet, C. J., Harris, W. E., \& McClure, R. D. 1995, ApJ, 450, 534

Driver, S. P., \& Phillips, S. 1996, ApJ, 469, 529
Driver, S. P., Phillips, S., Davies, J. I., Morgan, I., \& Disney, M. J. 1994a, MNRAS, 266, 155 1994b, MNRAS, 268, 393

Driver, S. P., Windhorst, R. A., \& Griffiths, R. E. 1995, ApJ, 453, 48

Eales, S. 1993, ApJ, 404, 51

Efstathiou, G., Ellis, R. S., \& Peterson, B. A. 1988, MNRAS, 232, 431

Ellis, R. S., Colless, M., Broadhurst, T., Heyl, J., \& Glazebrook, K. 1996, MNRAS, 280, 235

Gallego, J., Zamorano, J., Aragón-Salamanca, A., \& Rego, M. 1995, ApJ, 455, L1

Gardner, J. P., Sharples, R. M., Frenk, C. S., \& Carrasco, B. E. 1997, ApJ, 480, L99 
Glazebrook, K., Ellis, R., Santiago, B., \& Griffiths, R. 1995b, MNRAS, 275, L19

Heyl, J., Colless, M., Ellis, R. S., \& Broadhurst, T. 1997, MNRAS, 285, 613

Holtzman, J. A., et al. 1992, AJ, 103, 691

Hunsberger, S. D., Charlton, J. C., \& Zaritsky, D. 1996, ApJ, 462, 50

Kippenhahn, R., \& Weigert, A. 1990, Stellar Structure and Evolution (Berlin: Springer)

Koo, D. C., Gronwall, C., \& Bruzual, G. A. 1993, ApJ, 415, L21

Koo, D. C., \& Kron, R. G. 1992, ARA\&A, 30, 613

Lehnert, M. D., \& Heckman, T. M. 1996, ApJ, 472, 546

Leitherer, C., \& Heckman, T. M. 1995, ApJS, 96, 9

Lilly, S. J., Tresse, L., Hammer, F., Crampton, D., \& Le Fèvre, O. 1995, ApJ, 455,108

Lin, H., Kirshner, R. P., Shectman, S. A., Landy, S. D., Oemler, A., Tucker, D. L., \& Schechter, P. L. 1996, ApJ, 464, 60

Lin, H., Yee, H. K. C., Carlberg, R. G., \& Ellingson, E. E. 1997, ApJ, 475, 494

Lobo, C., Biviano, A., Durret, F., Gerbal, D., LeFèvre, O., Mazure, A., \& Slezak, E. 1996, A\&A, 317, 385

Loveday, J. 1997, ApJ, submitted

Loveday, J., Peterson, B. A., Efstathiou, G., \& Maddox, S. J. 1992, ApJ, 390, 338

Lutz, D. 1991, A\&A, 245, 31

Ma, C.-P., \& Bertschinger, E. 1994, ApJ, 434, L5

Marzke, R. O., Geller, M. J., Huchra, J. P., \& Corwin, H. G. 1994a, AJ, 108, 437

Marzke, R. O., Huchra, J. P., \& Geller, M. J. 1994b, ApJ, 428, 43
Metcalfe, N., Shanks, T., Fong, R., \& Roche, N. 1995, MNRAS, 273, 257

Meurer, G. R. 1995, Nature, 375, 742

Miley, G., Neugebauer, G., \& Soifer, B. T. 1985, ApJ, 293, L11

Mobasher, B., Sharples, R. M., \& Ellis, R. S. 1993, MNRAS, 263, 560

Press, W. H., \& Schechter, P. 1974, ApJ, 187, 425

Ratcliffe, A., Shanks, T., Parker, Q. A., \& Fong, R. 1997, MNRAS, in press

Renzini, A., \& Buzzoni, A. 1986, in Spectral Evolution in Galaxies, ed. C. Chiosi \& A. Renzini (Dordrecht: Reidel), 135

Rieke, G. H., \& Lebofsky, M. 1986, ApJ, 304, 326

Sandage, A., Binggeli, B., \& Tammann, G. A. 1985, AJ, 90, 1759

Santos, J. F. C., Bica, E., Dottori, H., Ortolani, S., \& Barbury, B. 1995, A\&A, 303,753

Saunders, W., Rowan-Robinson, M., Lawrence, A., Efstathiou, G., Kaiser, N., Ellis, R. S., \& Frenk, C. S. 1990, MNRAS, 242, 318

Schechter, P. 1976, ApJ, 203, 297

Scoville, N., \& Soifer, B. T. 1991, in Massive Stars in Starbursts, ed. C. Leitherer, N. R. Walborn, T. M. Heckman, \& C. A. Norman (Cambridge: Cambridge Univ. Press), 233

Silk, J., \& Wyse, R. G. 1993, Phys. Rep., 231, 293

Smail, I., Hogg, D. W., Yan, L., \& Cohen, J. G. 1995, ApJ, 449, L105

Soifer, B. T., Sanders, D. B., Madore, B. F., Neugebauer, G., Danielson, G. E., Elias, J. H., Lonsdale, C. J., \& Rice, W. L. 1987, ApJ, 320, 238

Telles, J. E. 1995, Ph.D. thesis, Univ. Cambridge

Treyer, M. A., \& Silk, J. 1994, ApJ, 436, L143

Whitmore, B. C., Schweizer, F., Leitherer, C., Borne, K., \& Robert, C. 1993, AJ, 106, 1354

Wilson, G., Smail, I., Ellis, R. S., \& Couch, W. J. 1997, MNRAS, 284, 915 